\title{
Optimierungspotenziale aufdecken
}

\section{Mit Hilfe des neuen Ressourcenchecks und des bereitgestellten Fragebogens können kleine und mittlere Unternehmen Einsparpotenziale im eigenen Beschichtungsverfahren abschätzen.}

Ob gasförmig, flüssig, gelöst oder fest: Je nach Beschichtungsverfahren verwenden Unternehmen in ihren Produktionsabläufen unterschiedliche Materialien. Der neue Ressourcencheck vom VDI Zentrum Ressourceneffizienz (VDI ZRE) zeigt auf, wo es Optimierungspotenziale gibt.

Beschichtungsverfahren selbst helfen bereits bei der Steigerung der Ressourceneffizienz: Beschichtete Bauteile profitieren, je nach Verfahren, zum Beispiel von einem verbesserten Korrosionsschutz und erreichen damit eine höhere Lebensdauer. Auch Werkzeuge wie Fräser oder Wendeschneidplatten werden häufig beschichtet, um den Verschleiß zu mindern und damit die Standzeit zu verlängern. Beschichtungsverfahren sind daher als Werterhaltung von großer Bedeutung für das verarbeitende Gewerbe und die Stahlbauindustrie.

\section{Einsparpotenziale abschätzen}

KMU aus dem Bereich der Beschichtungstechnik können mit den vom VDI ZRE entwickelten Arbeitsmitteln Einsparpotenzi- ale in ihren eigenen Verfahren abschätzen: Der Ressourcencheck Beschichten umfasst sowohl einen Fragebogen mit sechs Fragen zur Produktgestaltung, Material- und Energieeffizienz sowie Mitarbeitereinbindung als auch modular aufgebaute Checklisten. Im Ergebnis enthalten die Checklisten Methoden, Werkzeuge und Maßnahmen, die Anregungen zur Prozessoptimierung geben. Eine Einschätzung des Ressourceneffizienz-Potenzials erfolgt nach dem Ampelprinzip.

Darüber hinaus hat das VDI ZRE das aktuell verfügbare Wissen zu Ressourceneffizienz im Bereich Beschichten anhand einzelner Arbeitsschritte in einer Prozesskette systematisiert. Sie umfasst die Verfahren Emaillieren, Schmelztauchen, Wirbelsintern, Thermisches Spritzen sowie Pulverbeschichten und PVD/CVD; zu den weit verbreiteten Verfahren Lackieren und Galvanisieren sind eigene Prozessketten verfügbar.

In der Prozesskette zum Beschichten sind Informationen wie Gute-Praxis-Beispiele und aktuelle Forschungsprojekte integriert. KMU erfahren anhand von
Projektbeispielen und Videos, wie andere Unternehmen aus unterschiedlichen Technologiebereichen bereits ihre Ressourceneffizienz-Potenziale ausschöpfen. Zudem hilft eine interaktive Grafik den Unternehmen im Hinblick auf die eigenen Prozessabläufe, indem sie aufzeigt wie sich die betrieblichen Prozesse optimieren lassen. //

\section{Kontakt}

VDI Zentrum Ressourceneffizienz GmbH Berlin, Tel. 03027595060

zre-redaktion@vdi.de www.ressource-deutschland.de 